\title{
Half-duplex power beacon-assisted energy harvesting relaying networks: system performance analysis
}

\author{
Tan N. Nguyen ${ }^{1}$, Minh Tran ${ }^{2}$, Van-Duc Phan ${ }^{3}$, Hoang-Nam Nguyen ${ }^{4}$, Thanh-Long Nguyen 5 \\ ${ }^{1}$ Wireless Communications Research Group, Faculty of Electrical and Electronics Engineering, \\ Ton Duc Thang University, Vietnam \\ ${ }^{2}$ Optoelectronics Research Group, Faculty of Electrical and Electronics Engineering, \\ Ton Duc Thang University, Vietnam \\ ${ }^{3}$ Center of Excellence for Automation and Precision Mechanical Engineering, Nguyen Tat Thanh University, Vietnam \\ ${ }^{4}$ Modeling Evolutionary Algorithms Simulation and Artificial Intelligence, \\ Faculty of Electrical \& Electronics Engineering, Ton Duc Thang University, Vietnam \\ ${ }^{5}$ Center for Information Technology, Ho Chi Minh City University of Food Industry, Vietnam
}

\begin{tabular}{l} 
Article Info \\
\hline Article history: \\
Received Nov 11, 2018 \\
Revised Apr 6, 209 \\
Accepted Apr 18, 2019 \\
\hline Keywords: \\
Half-duplex (HD) \\
Outage probability (OP) \\
System throughput (ST) \\
Wireless energy harvesting \\
(EH) \\
\hline
\end{tabular}

\begin{abstract}
In this work, the half-duplex (HF) power beacon-assisted (PB) energy harvesting $(\mathrm{EH})$ relaying network, which consists of a source (S), Relay (R), destination (D) and a power beacon (PB) are introduced and investigated. Firstly, the analytical expressions of the system performance in term of outage probability (OP) and the system throughput (ST) are analyzed and derived in both amplify-and-forward (AF) and decode-and-forward (DF) modes. After that, we verify the correctness of the analytical analysis by using Monte-Carlo simulation in connection with the primary system parameters. From the numerical results, we can see that all the analytical and the simulation results are matched well with each other.
\end{abstract}

Copyright $\odot 2019$ Institute of Advanced Engineering and Science. All rights reserved.

\section{Corresponding Authors:}

Van-Duc Phan,

Center of Excellence for Automation and Precision Mechanical Engineering,

Nguyen Tat Thanh University,

Ho Chi Minh City, Vietnam.

Email:pvduc@ntt.edu.vn

Hoang-Nam Nguyen,

Modeling Evolutionary Algorithms Simulation and Artificial Intelligence,

Faculty of Electrical \& Electronics Engineering,

Ton Duc Thang University, Ho Chi Minh City, Vietnam.

Email: nguyenhoangnam@tdtu.edu.vn

\section{INTRODUCTION}

Energy harvesting $(\mathrm{EH})$ relay network, which uses a radio frequency $(\mathrm{RF})$ signal for wirelessly transferring power, has attracted much attention because of prolonging the lifetime of a wireless network. This solution can be obtained because RF signal can simultaneously transfer energy and information [1-10]. Nowadays, the system performance of EH relaying network has been studied in many studies. [11] investigated the full-duplex EH, the development of cooperative protocols for EH relaying network is fully studied in [12-13]. Furthermore, [14-15] introduce and investigate a "harvest-then-transmit" protocol for a multi-user relaying network. In all papers above, the relay (R) and the destination (D) nodes only receive energy from the source (S) or the access point nodes. In the trends to improving $\mathrm{EH}$ and information transmission (IT) processes in the wireless relay network, some researchers proposed the idea of deploying 
dedicated power beacon node $(\mathrm{PB})$. In the wireless relay network with using the $\mathrm{PB}$ node, $\mathrm{D}$ and $\mathrm{R}$ can receive energy both from $S$ and $\mathrm{PB}$ nodes [16-17]. The problem of energy harvesting is also encountered in directional MANET contexts involving high directive beamforming devices [18].

In this work, we introduce and investigate the system performance analysis of HD power beaconassisted EH relay network in both the amplifier-and-forward (AF) and decode-and-forward (DF) modes via the Raleigh fading channels. Firstly, the integral closed-form expression of the outage probability (OP) and system throughput (ST). After that, the analytical expressions are convinced by using Monte-Carlo simulation with helping Mat Lab software in both amplifier-and-forward (AF) and decode-and-forward (DF) modes. Finally, the numerical analysis can be demonstrated in connection with the primary system parameter.

\section{SYSTEM MODEL AND PERFORMANCE ANALYSIS}

Figure 1 illustrates the system model of the proposed system. In Figure 1, the information is transferred from $\mathrm{S}$ to $\mathrm{D}$ with helping of an intermediate $\mathrm{R}$. Here $\mathrm{S}$, and $\mathrm{R}$ are harvest energy from the PB node directly. In this model, all the block-fading channels are the Raleigh fading channels. Figure 2 illustrates the energy harvesting and information transmission processes. In this proposed system model, the node $\mathrm{PB}$ transfers the energy to $\mathrm{S}$ and $\mathrm{R}$ in $\alpha \mathrm{T}(\leq \alpha \leq 1)$. After that, $\mathrm{S}$ transfers the information to $\mathrm{R}$ in the next interval time $(1-\alpha) \mathrm{T} / 2$. Finally, the relay node $\mathrm{R}$ transfers the information to the destination node $\mathrm{D}$ in $(1-\alpha) \mathrm{T} / 2$.

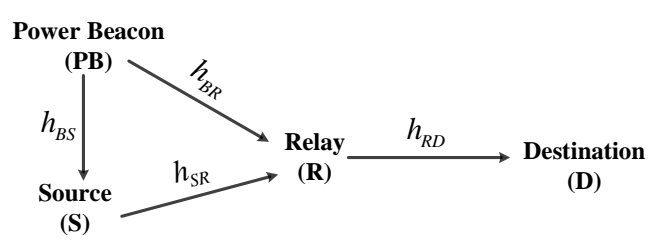

Figure 1. System model



Figure 2. EH and IT processes

\subsection{The amplifier and forward (AF) mode}

In $\alpha \mathrm{T}$, the PB node transfers energy to both source node $\mathrm{S}$ and relay node $\mathrm{R}$. Then the harvested energy at the source node $S$ can be calculated by

$$
E_{s}=\eta \alpha T P_{B}\left|h_{B S}\right|^{2}
$$

where $0<\eta<1$ is the energy conversion efficiency. From this energy, the average transmits power at $S$ can be formulated as

$$
P_{s}=\frac{E_{s}}{(1-\alpha) T / 2}=k P_{B}\left|h_{B S}\right|^{2}
$$

Similarity, the harvested energy at $\mathrm{R}$ is

$$
E_{r}=\eta \alpha T P_{B}\left|h_{B R}\right|^{2}
$$

Then the average transmits power at $\mathrm{R}$ is

$$
P_{r}=\frac{E_{r}}{(1-\alpha) T / 2}=k P_{B}\left|h_{B R}\right|^{2}
$$

where we denote that $k=\frac{2 \eta \alpha}{1-\alpha}$ 
After that, $\mathrm{S}$ transfers the information to $\mathrm{R}$. The received signal is

$$
y_{r}=h_{S R} x_{s}+n_{r}
$$

where $\mathrm{x}_{\mathrm{S}}$ is the transmission signal from $\mathrm{S}$.

In the next stage, $\mathrm{R}$ transfers the information, which is received from the source to $\mathrm{D}$ in the remaining interval time. The received signal at $\mathrm{D}$ is formulated as

$$
y_{d}=h_{R D} x_{r}+n_{d}
$$

where $\mathrm{n}_{\mathrm{r}}, \mathrm{n}_{\mathrm{d}}$ are the additive white Gaussian noise (AWGN) at $R, D$ with zero mean and variance $\mathrm{N}_{0}$, $\mathrm{E}\left[\left|x_{s}\right|^{2}\right]=P_{r}, \mathrm{E}\left[\left|x_{r}\right|^{2}\right]=P_{r}$, and $\mathrm{E}[\bullet]$ is expectation operator, $\mathrm{x}_{\mathrm{r}}$ is the transmission signal from $\mathrm{R}$.

In the AF mode, we can calculate the amplifier factor as

$$
\beta=\frac{x_{r}}{y_{r}}=\sqrt{\frac{P_{r}}{P_{s}\left|h_{S R}\right|^{2}+N_{0}}}
$$

From (6) and (7), the received signal at D is

$$
y_{d}=h_{R D} \beta y_{r}+n_{d}=h_{R D} \beta\left[h_{S R} x_{s}+n_{r}\right]+n_{d}=\underbrace{h_{S R} h_{R D} \beta x_{s}}_{\text {signal }}+\underbrace{h_{R D} \beta n_{r}+n_{d}}_{\text {noise }}
$$

Using (8), we can calculate the signal to noise ratio (SRN) at D by

$$
\gamma_{e 2 e}=\frac{\beta^{2}\left|h_{S R}\right|^{2}\left|h_{R D}\right|^{2} P_{s}}{\left|h_{R D}\right|^{2} \beta^{2} N_{0}+N_{0}}=\frac{\left|h_{S R}\right|^{2}\left|h_{R D}\right|^{2} P_{s}}{\left|h_{R D}\right|^{2} N_{0}+\frac{N_{0}\left(P_{s}\left|h_{S R}\right|^{2}+N_{0}\right)}{P_{r}}}
$$

The equation (10) is reformulated by using $\mathrm{N}_{0}<<\mathrm{P}_{\mathrm{r}}$

$$
\gamma_{e 2 e}=\frac{\left|h_{S R}\right|^{2}\left|h_{R D}\right|^{2} P_{s} P_{r}}{\left|h_{R D}\right|^{2} P_{r} N_{0}+N_{0} P_{s}\left|h_{S R}\right|^{2}}
$$

Combining with (2),(4) and substituting into (11), SNR is

$$
\begin{aligned}
& \qquad \gamma_{e 2 e}=\frac{k P_{B}\left|h_{S R}\right|^{2}\left|h_{R D}\right|^{2}\left|h_{B R}\right|^{2}\left|h_{B S}\right|^{2}}{\left|h_{R D}\right|^{2}\left|h_{B R}\right|^{2} N_{0}+N_{0}\left|h_{B S}\right|^{2}\left|h_{S R}\right|^{2}}=\frac{k \gamma_{0} X Y}{X+Y} \\
& \text { where we denote } \gamma_{0}=\frac{P_{B}}{N_{0}}, X=\left|h_{R D}\right|^{2}\left|h_{B R}\right|^{2}, Y=\left|h_{S R}\right|^{2}\left|h_{B S}\right|^{2} .
\end{aligned}
$$

For more analysis, utilizing the result in [19], the c.d.f. of $X$ and $Y$ is

$$
\begin{aligned}
& F_{X}(x)=1-2 \sqrt{\lambda_{R D} \lambda_{B R} x} K_{1}\left(2 \sqrt{\lambda_{R D} \lambda_{B R} x}\right) \\
& F_{Y}(y)=1-2 \sqrt{\lambda_{S R} \lambda_{B S} y} K_{1}\left(2 \sqrt{\lambda_{S R} \lambda_{B S} y}\right)
\end{aligned}
$$


OP of the proposed system is

$$
\begin{aligned}
& P_{\text {out }}^{A F}=\operatorname{Pr}\left(\gamma_{e 2 e}<\gamma_{t h}\right)=\operatorname{Pr}\left(\frac{k \gamma_{0} X Y}{X+Y}<\gamma_{t h}\right)=\operatorname{Pr}\left(X\left[k \gamma_{0} Y-\gamma_{t h}\right]<\gamma_{t h} Y\right) \\
& =\operatorname{Pr}\left\{\begin{array}{cc}
X<\frac{\gamma_{t h} Y}{k \gamma_{0} Y-\gamma_{t h}}, Y \geq \frac{\gamma_{t h}}{k \gamma_{0}} \\
1 \quad, Y<\frac{\gamma_{t h}}{k \gamma_{0}}
\end{array}\right.
\end{aligned}
$$

where $\gamma_{t h}=2^{R}-1$ is a threshold, and $\mathrm{R}$ is source rate.

Proposition 1: OP of the proposed system is given by

$$
P_{\text {out }}^{A F}=1-4 \lambda_{S R} \lambda_{B S} \int_{\frac{\gamma_{\text {th }}}{k \gamma_{0}}}^{\infty} \sqrt{\frac{\lambda_{R D} \lambda_{B R} \gamma_{t h} y}{k \gamma_{0} y-\gamma_{t h}}} K_{0}\left(2 \sqrt{\lambda_{S R} \lambda_{B S} y}\right) K_{1}\left(2 \sqrt{\frac{\lambda_{R D} \lambda_{B R} \gamma_{t h} y}{k \gamma_{0} y-\gamma_{t h}}}\right) d y
$$

Proof: See Appendix A.

Finally, The system throughput at D is formulated as

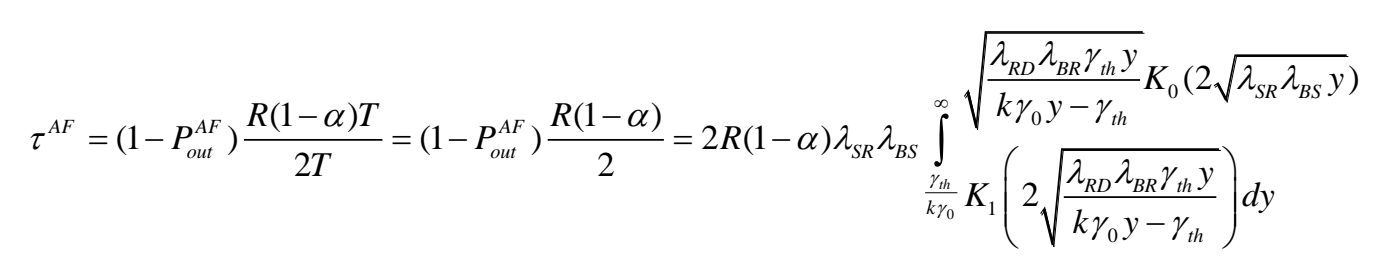

\subsection{The decode and forward (DF) mode}

From (1) and (2) we have the SNR for the DF mode:

$$
\begin{aligned}
& S N R_{1}=\frac{P_{s}\left|h_{S R}\right|^{2}}{N_{0}}=\frac{k P_{B}\left|h_{B S}\right|^{2}\left|h_{S R}\right|^{2}}{N_{0}}=k \gamma_{0} Y \\
& S N R_{2}=\frac{P_{r}\left|h_{R D}\right|^{2}}{N_{0}}=\frac{k P_{B}\left|h_{B R}\right|^{2}\left|h_{R D}\right|^{2}}{N_{0}}=k \gamma_{0} X
\end{aligned}
$$

Furthermore, OP of the proposed system is

$$
P_{\text {out }}^{D F}=\operatorname{Pr}\left(\min \left[S N R_{1}, S N R_{2}\right]<\gamma_{\text {th }}\right)=\operatorname{Pr}\left\{\min \left[k \gamma_{0} Y, k \gamma_{0} X\right]<\gamma_{\text {th }}\right\}
$$

Proposition 2: The outage probability at the destination node of the proposed system is given by:

$$
P_{\text {out }}^{D F}=1-4 \frac{\gamma_{\text {th }}}{k \gamma_{0}} \sqrt{\lambda_{R D} \lambda_{B R} \lambda_{S R} \lambda_{B S}} K_{1}\left(2 \sqrt{\frac{\lambda_{R D} \lambda_{B R} \gamma_{t h}}{k \gamma_{0}}}\right) K_{1}\left(2 \sqrt{\frac{\lambda_{S R} \lambda_{B S} \gamma_{t h}}{k \gamma_{0}}}\right)
$$

Proof: See Appendix B.

Finally, the throughput $\tau$ at $\mathrm{D}$ is given by:

$$
\tau^{D F}=\left(1-P_{\text {out }}^{D F}\right) \frac{R(1-\alpha)}{2}=2 R(1-\alpha) \frac{\gamma_{t h}}{k \gamma_{0}} \sqrt{\lambda_{R D} \lambda_{B R} \lambda_{S R} \lambda_{B S}} K_{1}\left(2 \sqrt{\frac{\lambda_{R D} \lambda_{B R} \gamma_{t h}}{k \gamma_{0}}}\right) K_{1}\left(2 \sqrt{\frac{\lambda_{S R} \lambda_{B S} \gamma_{t h}}{k \gamma_{0}}}\right)
$$




\section{RESULTS AND DISCUSSION}

We use the Monte Carlo simulation to verify the correctness of the analytical expression of the OP and ST in the above section in the connection of the primary parameters of the proposed system. Other simulation parameters are listed in Table 1.

Table 1. Simulation parameters

\begin{tabular}{lll}
\hline Symbol & Name & Values \\
\hline$\eta$ & Energy harvesting efficiency & 0.7 \\
$\lambda_{B S}$ & Mean of $\left|h_{B S}\right|^{2}$ & 0.5 \\
$\lambda_{B R}$ & Mean of $\left|h_{B R}\right|^{2}$ & 0.5 \\
$\lambda_{S R}$ & Mean of $\left|h_{S R}\right|^{2}$ & 0.5 \\
$\lambda_{R D}$ & Mean of $\left|h_{R D}\right|^{2}$ & \\
$\gamma_{t h}$ & SNR threshold & 0.5 \\
$\mathrm{P}_{\mathrm{B}} / \mathrm{N}_{0}$ & Source power to noise ratio & 7 \\
$\mathrm{R}$ & Source rate & $0-20 \mathrm{~dB}$ \\
\hline & & $1.5 \mathrm{bit} / \mathrm{s} / \mathrm{Hz}$ \\
\hline
\end{tabular}

Figure 3 and Figure 4 plot the curves of OP and ST versus $\alpha$ for both AF and DF modes. It is shown in Figure 3 and Figure 4 that $\mathrm{P}_{\mathrm{B}} / \mathrm{N}_{0}$ at 10 and $20 \mathrm{~dB}$, and $\alpha$ varies from 0 to 1 . We can see from the results that OP has a decrease when $\alpha$ increase from 0 to 1 as shown Figure 3. Moreover, Figure 4 shows that the throughput increases in the first stage when $\alpha$ increases from 0 to the optimal value. After that, the system throughput has a significant decrease while $\alpha$ varies from optimal value to 1 . In both Figure 3 and Figure 4, the analytical and simulation results agree very well with each other.

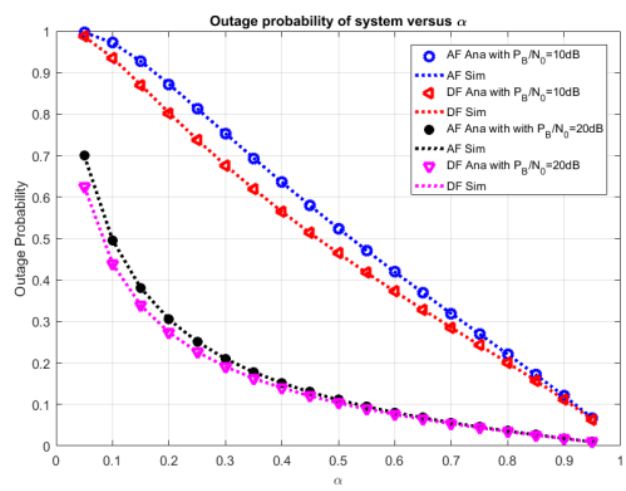

Figure 3. Outage probability versus $\alpha$

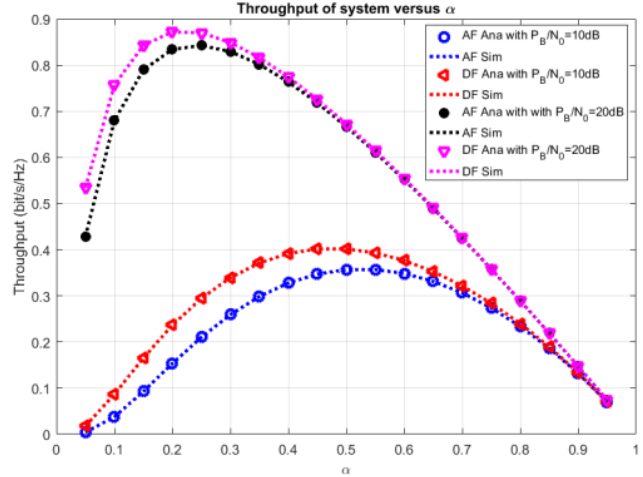

Figure 4. Throughput versus $\alpha$

Figure 5 and Figure 6 illustrate the effect of $\eta$ on OP and ST of the model system. Here we set $\alpha=0.5$ and $\mathrm{P}_{\mathrm{B}} / \mathrm{N}_{0}$ at 10 and $20 \mathrm{~dB}$, respectively. From the results, OP decreases while $\eta$ increases from 0 to 1 as shown in Figure 5. In contrast, ST has a considerable improvement when $\eta$ increases from 0 to 1 as shown in Figure 6. For both cases, the analytical simulation results are the same values. Moreover, OP and ST versus R are shown in Figure 7 and Figure 8, respectively. Similarity, we set $\mathrm{P}_{\mathrm{B}} / \mathrm{N}_{0}$ at 10 and $\alpha$ at $0.2,0.8$. From Figure 7 we see that OP increases while R varies from 1 to 8 . In contrast, ST increase in the first interval $\mathrm{R}$ to optimal value then has a huge decrease as shown in Figure 8. Furthermore, Figure 10 shows the optimal time switching factor of the proposed system at $\mathrm{R}=1$ and $3 \mathrm{bps}$. In all figures, the simulation and analytical results agree with each other. 


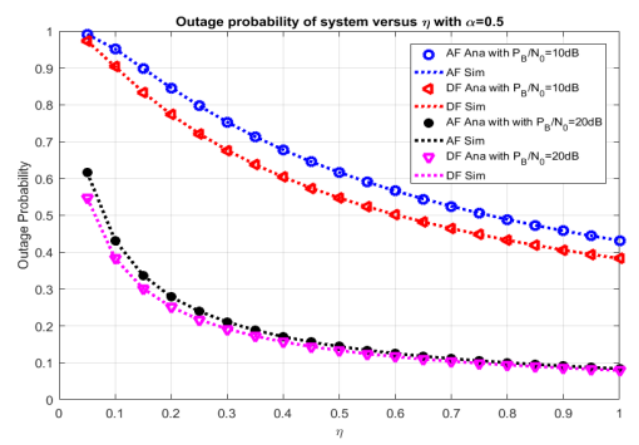

Figure 5. Outage probability versus $\eta$

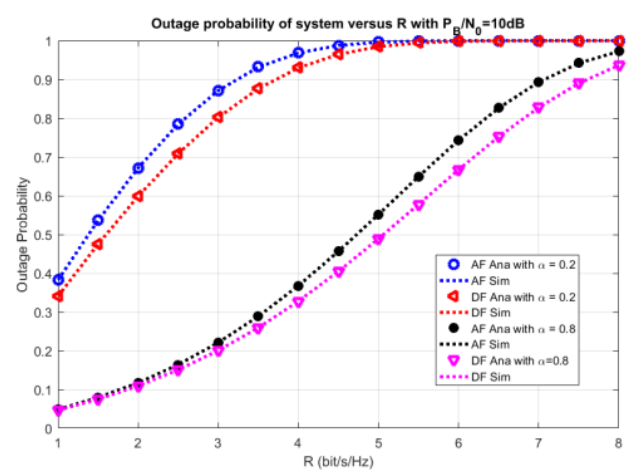

Figure 7. Outage probability versus R

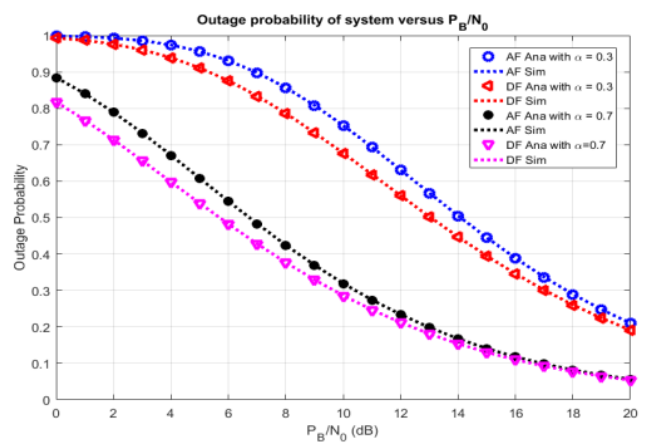

Figure 9. Outage probability versus PB/N0

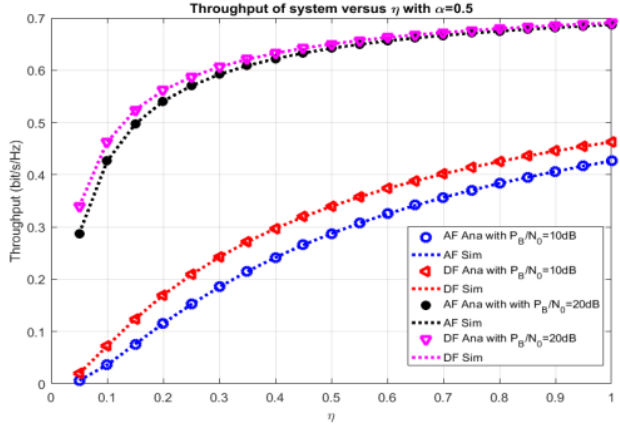

Figure 6. Throughput versus $\eta$



Figure 8. Throughput versus R

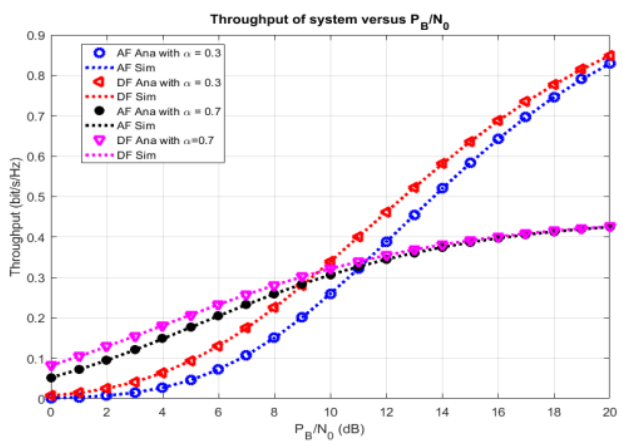

Figure 10. Throughput versus PB/N0

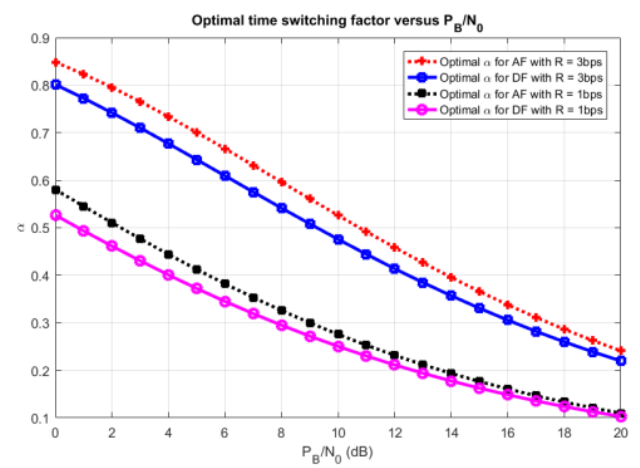

Figure 11. Optimal time switching factor versus PB/N0 


\section{CONCLUSION}

In this paper, we investigate the HD PB EH relay network in both AF and DF modes. We derive the closed-form expressions of OP and ST of the model system. Moreover, the analytical analysis is convinced totally by the Monte Carlo simulation. Also, the optimal time switching factor is investigated. All the analytical and simulation are the same with the primary system parameters. The results can be considered as the recommendation for EH relaying network research.

\section{REFERENCES}

[1] S. Bi, et al., "Wireless powered communication: Opportunities and challenges," IEEE Communications Magazine, vol/issue: 53(4), pp. 117-125, 2015.

[2] D. Niyato, et al., "Wireless Powered Communication Networks: Research Directions and Technological Approaches," IEEE Wireless Communications, pp. 2-11, 2017.

[3] A. A. Nasir, et al., "Relaying Protocols for Wireless Energy Harvesting and Information Processing," IEEE Transactions on Wireless Communications, vol/issue: 12(7), pp. 3622-3636, 2013.

[4] R. Atallah, et al., "Energy harvesting in vehicular networks: A contemporary survey," IEEE Wireless Communications, vol/issue: 23(2), pp. 70-77, 2016.

[5] H. Yu, et al., "What is 5G? Emerging 5G Mobile Services and Network Requirements," Sustainability, vol/issue: 9(10), pp. 1848, 2017.

[6] K. Huang and V. K. Lau, "Enabling Wireless Power Transfer in Cellular Networks: Architecture, Modeling and Deployment," IEEE Transactions on Wireless Communications, vol/issue: 13(2), pp. 902-912, 2014.

[7] X. Zhou, et al., "Wireless Information and Power Transfer: Architecture Design and Rate-Energy Tradeoff," IEEE Transactions on Communications, vol/issue: 61(11), pp. 4754-4767, 2013.

[8] F. D. Rango and M. Tropea, "Energy saving and load balancing in wireless ad hoc networks through ant-based routing," Performance Evaluation of Computer \& Telecommunication Systems, 2009. SPECTS 2009. International Symposium on, vol. 41, pp. 117-124, 2009.

[9] F. D. Rango and M. Tropea, "Swarm intelligence based energy saving and load balancing in wireless ad hoc networks," in Proceedings of the 2009 workshop on Bio-inspired algorithms for distributed systems, pp. 77-84, 2009.

[10] F. D. Rango, et al., "MDMC: A WSN cooperative protocol for Minimizing the Data Distortion," Proceedings of the 2018 11th IFIP Wireless and Mobile Networking Conference, WMNC, 2018.

[11] H. Ju and R. Zhang, "Optimal Resource Allocation in Full-Duplex Wireless-Powered Communication Network," IEEE Transactions on Communications, vol/issue: 62(10), pp. 3528-3540, 2014.

[12] Q. Yao, et al., "Adaptive harvest-then-cooperate: Delay-aware wireless powered communication networks," 2016 IEEE 17th International Workshop on Signal Processing Advances in Wireless Communications (SPAWC), 2016.

[13] Y. Gu, et al., "An adaptive transmission protocol for wireless-powered cooperative communications," 2015 IEEE International Conference on Communications (ICC), 2015.

[14] $\mathrm{H}$. Ju and R. Zhang, "Throughput Maximization in Wireless Powered Communication Networks," IEEE Transactions on Wireless Communications, vol/issue: 13(1), pp. 418-428, 2014.

[15] K. Huang and V. K. Lau, "Enabling Wireless Power Transfer in Cellular Networks: Architecture, Modeling and Deployment," IEEE Transactions on Wireless Communications, vol/issue: 13(2), pp. 902-912, 2014

[16] Y. Ma, et al., "Distributed resource allocation for power beacon-assisted wireless-powered communications," 2015 IEEE International Conference on Communications (ICC), 2015.

[17] J. Park, et al., "Energy Beamforming for Wireless Power Transfer in MISO Heterogeneous Network With Power Beacon," IEEE Communications Letters, vol/issue: 21(5), pp. 1163-1166, 2017.

[18] V. Inzillo, et al., "An Adaptive Beamforming Time with Round-Robin MAC Algorithm for Reducing Energy Consumption in MANET," Journal of Sensor and Actuator Networks, vol/issue: 7(4), pp. 50, 2018.

\section{APPENDIX A}

From (15), we have:

$$
\begin{aligned}
& P_{\text {out }}^{A F}=\int_{0}^{\frac{\gamma_{t h}}{k \gamma_{0}}} f_{Y}(y) d y+\int_{\frac{\gamma_{t h}}{k \gamma_{0}}}^{\infty} f_{Y}(y) d y \int_{0}^{\frac{\gamma_{h} Y}{k \gamma_{0} Y-\gamma_{t h}}} f_{X}(x) d x \\
& =\int_{0}^{\frac{\gamma_{t h}}{k \gamma_{0}}} f_{Y}(y) d y+\int_{\frac{\gamma_{\text {th }}}{k \gamma_{0}}}^{\infty} f_{Y}(y) d y\left\{1-2 \sqrt{\frac{\lambda_{R D} \lambda_{B R} \gamma_{t h} Y}{k \gamma_{0} Y-\gamma_{t h}}} K_{1}\left(2 \sqrt{\frac{\lambda_{R D} \lambda_{B R} \gamma_{t h} Y}{k \gamma_{0} Y-\gamma_{t h}}}\right)\right\} \\
& P_{\text {out }}^{A F}=1-2 \int_{\frac{\gamma_{t h}}{k \gamma_{0}}}^{\infty} \sqrt{\frac{\lambda_{R D} \lambda_{B R} \gamma_{t h} Y}{k \gamma_{0} Y-\gamma_{t h}}} K_{1}\left(2 \sqrt{\frac{\lambda_{R D} \lambda_{B R} \gamma_{t h} Y}{k \gamma_{0} Y-\gamma_{t h}}}\right) f_{Y}(y) d y
\end{aligned}
$$

Half-duplex power beacon-assisted energy harvesting relaying networks: system ... (Tan N. Nguyen) 
So, we can compute $f_{Y}(y)$ by using equation $[8.486,18]$ in [22]

$$
\begin{aligned}
& \frac{d}{d z}\left(z^{v} K_{v}(z)\right)=-z^{v} K_{v-1}(z) \\
& f_{Y}(y)=\frac{d\left\{F_{Y}(y)\right\}}{d y}=2 \lambda_{S R} \lambda_{B S} K_{0}\left(2 \sqrt{\lambda_{S R} \lambda_{B S} y}\right)
\end{aligned}
$$

Combine (A3), (A4), OP is obtained as in (16).

\section{APPENDIX B}

$$
\begin{aligned}
& P_{\text {out }}^{D F}=1-\operatorname{Pr}\left(X \geq \frac{\gamma_{\text {th }}}{k \gamma_{0}}, Y \geq \frac{\gamma_{\text {th }}}{k \gamma_{0}}\right)=1-\operatorname{Pr}\left(X \geq \frac{\gamma_{\text {th }}}{k \gamma_{0}}\right) \operatorname{Pr}\left(Y \geq \frac{\gamma_{\text {th }}}{k \gamma_{0}}\right) \\
& \operatorname{Pr}\left(X \geq \frac{\gamma_{t h}}{k \gamma_{0}}\right)=1-\operatorname{Pr}\left(X<\frac{\gamma_{\text {th }}}{k \gamma_{0}}\right)=1-F_{X}\left(\frac{\gamma_{t h}}{k \gamma_{0}}\right)
\end{aligned}
$$

From (9) we obtain:

$$
\operatorname{Pr}\left(X \geq \frac{\gamma_{t h}}{k \gamma_{0}}\right)=2 \sqrt{\frac{\lambda_{R D} \lambda_{B R} \gamma_{t h}}{k \gamma_{0}}} K_{1}\left(2 \sqrt{\frac{\lambda_{R D} \lambda_{B R} \gamma_{t h}}{k \gamma_{0}}}\right)
$$

Similar as above, we can compute:

$$
\operatorname{Pr}\left(Y \geq \frac{\gamma_{t h}}{k \gamma_{0}}\right)=2 \sqrt{\frac{\lambda_{S R} \lambda_{B S} \gamma_{t h}}{k \gamma_{0}}} K_{1}\left(2 \sqrt{\frac{\lambda_{S R} \lambda_{B S} \gamma_{t h}}{k \gamma_{0}}}\right)
$$

From (B4), (20) is demonstrated. 